\section{CDCA7 is a critical mediator of lymphomagenesis that selectively regulates anchorage-independent growth}

\author{
Raúl Jiménez-P., ${ }^{1}$ Carla Martín-Cortázar, ${ }^{1}$ Omar Kourani, ${ }^{1}$ Yuri Chiodo, ${ }^{1}$ \\ Raul Cordoba, ${ }^{2, \dagger}$ María Purificación Domínguez-Franjo, ${ }^{3,+}$ \\ Juan Miguel Redondo, ${ }^{4,5}$ Teresa Iglesias ${ }^{6,7}$ and Miguel R. Campanero ${ }^{1,5}$
}

${ }^{1}$ Department of Cancer Biology, Instituto de Investigaciones Biomédicas Alberto Sols, CSIC-UAM; '² Department of Hematology, University Hospital Infanta Sofía, San Sebastián de los Reyes; ${ }^{3}$ Department of Pathology, University Hospital Infanta Sofía, San Sebastián de los Reyes; ${ }^{4}$ Department of Vascular Biology and Inflammation, Centro Nacional de Investigaciones Cardiovasculares, (CNIC); ${ }^{5} \mathrm{CIBERCV}$, Spain; ${ }^{6}$ Department of Endocrine and Nervous Systems Pathophysiology, Instituto de Investigaciones Biomédicas Alberto Sols, CSIC-UAM, Madrid and ${ }^{7}$ Centro de Investigación Biomédica en Red sobre Enfermedades Neurodegenerativas (CIBERNED), Madrid, Spain

†Present address: Lymphoma Unit, Department of Hematology, Fundacion Jimenez Diaz University Hospital, Health Research Institute IIS-FJD, Madrid, Spain

†Present address: Department of Pathology, Hospital Rey Juan Carlos, Mostoles, Madrid, Spain

\section{ABSTRACT}

T umor formation involves the acquisition of numerous capacities along the progression from a normal cell into a malignant cell, including limitless proliferation (immortalization) and anchorageindependent growth, a capacity that correlates extremely well with tumorigenesis. Great efforts have been made to uncover genes involved in tumor formation, but most genes identified participate in processes related to cell proliferation. Accordingly, therapies targeting these genes also affect the proliferation of normal cells. To identify potential targets for therapeutic intervention more specific to tumor cells, we looked for genes implicated in the acquisition of anchorage-independent growth and in vivo tumorigenesis capacity. A transcriptomic analysis identified CDCA7 as a candidate gene. Indeed, CDCA7 protein was upregulated in Burkitt's lymphoma cell lines and human tumor biopsy specimens relative to control cell lines and tissues, respectively. CDCA7 levels were also markedly elevated in numerous $\mathrm{T}$ and B-lymphoid tumor cell lines. While CDCA7 was not required for anchorage-dependent growth of normal fibroblasts or non-malignant lymphocytes, it was essential but not sufficient for anchorage-independent growth of lymphoid tumor cells and for lymphomagenesis. These data suggest that therapies aimed at inhibiting CDCA7 expression or function might significantly decrease the growth of lymphoid tumors.

\section{Introduction}

Most side effects of current therapies for cancer treatment are derived from their toxicity on actively proliferating normal cells, such as hematopoietic progenitors. These toxic effects likely occur because the targets for these therapies are also crucial for the proliferation of normal cells. The development of therapies more selective for tumor cells might be facilitated by the identification of genes involved in properties specific of these cells.

Along the transformation of a normal cell into a highly malignant derivative, cells acquire numerous traits, including the ability to sustain chronic proliferation. ${ }^{1,2}$ Although immortalization is a fundamental trait of cancer cells, it is insufficient to promote malignant growth. NIH-3T3 fibroblasts, for instance, display replicative immortality but are not tumorigenic and display in vitro growth characteristics of
Haematologica 2018

Volume 103(10):1669-1678

\section{Correspondence:}

mcampanero@iib.uam.es

Received: January 18, 2018

Accepted: June 4, 2018

Pre-published: June 7, 2018.

doi:10.3324/haematol.2018.188961

Check the online version for the most updated information on this article, online supplements, and information on authorship \& disclosures: www.haematologica.org/content/103/10/1669

\section{(C)2018 Ferrata Storti Foundation}

Material published in Haematologica is covered by copyright. All rights are reserved to the Ferrata Storti Foundation. Use of published material is allowed under the following terms and conditions:

https://creativecommons.org/licenses/by-nc/4.0/legalcode. Copies of published material are allowed for personal or internal use. Sharing published material for non-commercial purposes is subject to the following conditions: https://creativecommons. org//icenses/by-nc/4.0/legalcode, sect. 3. Reproducing and sharing published material for commercial purposes is not allowed without permission in writing from the publisher. 
non-transformed cells. ${ }^{3}$ Epstein-Barr virus (EBV) infection of normal lymphocytes generates immortalized lymphoblastoid B-cell lines (LCLs) unable to form tumors in immunodeficient mice but capable to replicate indefinitely in liquid culture. ${ }^{4}$ In contrast, cell lines derived from Burkitt's lymphoma (BL), a B-lymphocyte tumor strongly associated with EBV in some regions of Africa, ${ }^{5}$ not only display replicative immortality, but are also tumorigenic in immunodeficient mice. ${ }^{4}$

Another trait of tumor cells is their capacity to replicate and grow independently of their attachment to a rigid surface. Growth of normal tissue cells requires the signals transmitted by plasma membrane receptors that bind extracellular matrix components and transmembrane proteins from neighboring cells of the proper microenvironment. Most normal tissue cells are not viable when suspended in liquid or soft medium, and require adhesion to the surface of a culture vessel. Similarly, immortal, but non-tumoral cells, including NIH-3T3 fibroblasts and LCLs, cannot grow in semi-solid media such as soft agar, ${ }^{4,67}$ and are considered as anchorage-dependent. On the contrary, tumor cells do not need to adhere to a rigid surface for growth and are said to be anchorage-independent. ${ }^{6}$ Numerous genes that mediate tumorigenesis have been identified, but very limited information is available regarding genes that specifically mediate anchorage-independent growth. While anchorage-dependence has been extensively studied in fibroblasts and epithelial cells, it is unknown whether normal lymphoid cells require anchorage for proliferation. Soft agar not only limits cell binding to the culture vessel surface but also intercellular interactions. The incapacity of LCLs to grow in soft agar could therefore be attributed to lack of anchorage to a rigid substrate or to neighboring cells. It should be noted that normal lymphoid cells proliferate only in lymphoid organs in vivo, in close contact with the extracellular matrix and with other cells; with appropriate stimuli, these cells can also proliferate in vitro under conditions that permit their attachment to the culture vessel surface and to other cells.

MYC deregulation is one of the most common aberrations in human tumors. The characteristic genetic marker of $\mathrm{BL}$ cells is a reciprocal translocation involving the $M Y C$ gene and one of three immunoglobulin gene loci that leads to deregulated MYC expression. ${ }^{8}$ MYC encodes a transcription factor and chromatin remodeler that regulates the expression of numerous genes involved in various cellular processes, including cell differentiation, proliferation, and apoptosis. ${ }^{914}$ Tumorigenesis by MYC (also known as C-MYC) can take place as a consequence of its overexpression, even in the absence of mutations in its coding region. ${ }^{15} \mathrm{E} \mu$-Myc transgenic mice, where Myc overexpression is targeted to B lymphocytes, give rise to lymphomas, but only after a mean latency period of about 6 months, these lymphomas being monoclonal. ${ }^{16}$ In addition, MYC overexpression in normal cells either arrests them in the G2 phase of the cell cycle ${ }^{17}$ or induces apoptosis. ${ }^{18}$ Together, these results suggest that MYC alone cannot elicit tumoral transformation of normal cells and that additional factors might cooperate with MYC in tumorigenesis.

MYC regulates about 15 percent of the genes in the human genome, and it is expected that some of them participate in tumor formation. However, it remains unknown which of these genes are critical for MYCinduced transformation. CDCA7, also named JPO1, is a
MYC-induced gen ${ }^{19}$ whose mRNA is deregulated in several tumor types relative to the corresponding non-proliferative control tissues..$^{20}$ Little is known about the molecular function of CDCA7. Phosphorylation by AKT regulates CDCA7 subcellular localization and its association with MYC. ${ }^{21}$ There are two alternatively spliced CDCA7 isoforms which contain a zinc finger domain at the C-terminus $^{22,23}$ and associate with the Helicase, Lymphoid-specific (HELLS) SNF2 family member. ${ }^{24}$ In fact, CDCA7 is required for nucleosome remodeling by HELLS and for DNA methylation maintenance. ${ }^{23,24}$ However, no functional differences between CDCA7 isoforms have been reported.

CDCA7 overexpression in tumors could potentially be just a consequence of the presence of more cycling cells in the tumor tissues. Indeed, its expression is induced in the S-phase of the cell cycle ${ }^{25}$ and it is a transcriptional target not only of MYC ${ }^{19}$ but also of E2F1-E2F4, ${ }^{22}$ factors that are active exclusively in proliferating cells. Alternatively, CDCA7 could potentially play a causative role in tumorigenesis. In this regard, CDCA7 has been assumed to participate in neoplastic transformation of $B$ cells (http://www.uniprot.org/uniprot/Q9BWT1; https://Www. ncbi.nlm.nih.gov/gene/83879), in spite of insufficient evidence supporting this assumption. Indeed, previous observations showed that forced expression of CDCA7 barely increased colony formation in a B-cell line already capable of growing in soft agar. ${ }^{19}$ In addition, transgenic mice overexpressing CDCA7 in the B-cell compartment generated lymphoid malignancies as frequently as control mice (4 out of 45 transgenic mice vs. 3 out of 28 control mice). ${ }^{20}$ Moreover, CDCA7 forced expression inhibited the induction of anchorage-independent growth induced by MYC in immortal fibroblasts. ${ }^{21}$ Therefore, a tumorigenic role for CDCA7 has not been experimentally demonstrated and it is, at minimum, controversial.

The design of anti-tumor therapies that do not affect normal cells has proved to be a very difficult task. To identify potential therapeutic targets specific to tumor cells, we used a transcriptomic approach looking for genes specifically involved in anchorage-independent growth, a capacity that correlates extremely well with tumorigenesis. Here we show that CDCA7 was one of the most significantly up-regulated genes, that its encoded protein is overexpressed in lymphoid tumors, and that its silencing greatly impairs lymphomagenesis without inhibiting the proliferation of normal cells.

\section{Methods}

Detailed Methods can be found in the Online Supplementary Appendix.

\section{Patients}

Cases consisted of existing de-identified anonymous biopsy specimens obtained from Hospital Infanta Sofia and from the Spanish Tumor Bank Network in Centro Nacional de Investigaciones Oncológicas (Madrid, Spain). The study was approved by the CSIC Ethics Committee and by the Ethics Committee of Hospital Universitario La Paz (ref. HULP: PI-1658).

\section{Microarray Experiment Data Analysis}

RNA from BL and LCL cell lines was extracted and labelled as described. ${ }^{26}$ The RNA from X50-7, IB-4, and Dana LCL cell lines 
A

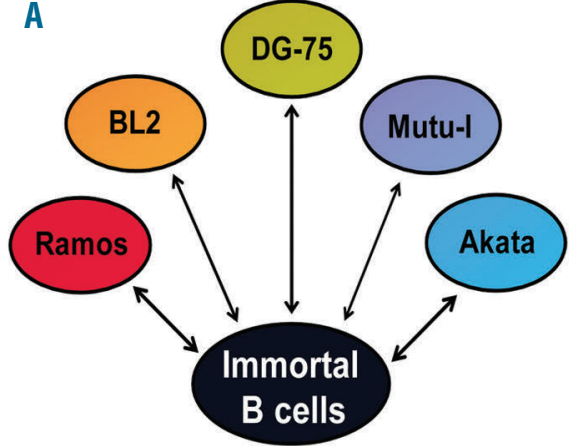

B

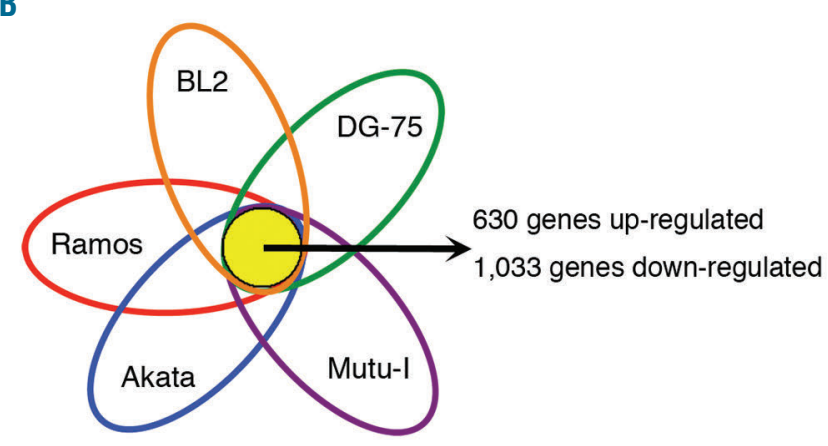

C

\begin{tabular}{|l|l|l|l|l|}
\hline Name & Fold & adj.P.Val. & geneid_EnsembI & Biological process \\
\hline BMP7 & 17.16 & 0.0007 & ENSG00000101144 & Chondrogenesis, osteogenesis \\
\hline ID3 & 8.26 & 0.0022 & ENSG00000117318 & Transcription regulation \\
\hline SLC2A5 & 7.63 & 0.0025 & ENSG00000142583 & Glucose/Fructose transport \\
\hline CUX1 & 6.56 & 0.0026 & ENSG00000160967 & Development \\
\hline CDCA7 & 5.90 & 0.0024 & ENSG00000144354 & Transcription regulation \\
\hline RBFOX2 & 3.96 & 0.0022 & ENSG00000100320 & RNA splicing \\
\hline DHFR & 3.14 & 0.0017 & ENSG00000168129 & Nucleotide biosynthesis \\
\hline
\end{tabular}

Figure 1. The expression profiles between BL and LCL are different. A. Experimental design. The expression profile of 5 BL cell lines (BL2, Ramos, DG-75, Akata, and Mutu-I) was compared with that of a pool of LCL lines (X50-7, IB4 and Dana). Each BL cell line was considered as a biological replica for data analysis. B. Venn diagram showing overlapping of genes significantly regulated relative to the pool of LCL. The total number of overlapping up- and down-regulated genes is shown. C. Genes up- (59 genes) or down-regulated (425 genes) >3-fold were selected and the most statistically significant genes (TOP 100) were further selected. The only genes among the TOP 100 that were up-regulated in $\mathrm{BL}$ cell lines are shown indicating their relative expression (Fold), adjusted $P$ value, Ensemble gene identification number, and the biological process in which these genes might be involved.

was pooled and used as a universal control for the whole experiment. The RNA from DG-75, Ramos, BL2, Mutu-I, and Akata BL cell lines was mixed with an equal amount of universal control pool and processed for microarray analysis using 22K-oligo microarrays (CapitalBio Corporation). Genes with similar expression within BL cell lines were ranked according to their $P$-value for differential expression (adj. $P$ value $<0.05$ ). Gene expression data are available at http://www.ncbi.nlm.nih.gov/projects/geo/ (accession number GSE41865).

\section{Northern blotting and quantitative PCR analysis}

Northern blotting was performed as described ${ }^{27}$ using 32P-labeled 5' CDCA7-2 and ACTG DNA fragments. Real-time quantitative RT-PCR ( $q-P C R$ ) was performed using TaqMan Gene Expression Assays (ThermoFischer Scientific) specific for both isoforms of human CDCA7 (Hs00230589_m1), CDCA7-1 (Hs00912235_m1), CDCA7-2 (Hs00914361_m1), or TBP (Hs00427621_m1).

\section{Cell Transfection, lentivirus production and cell transduction}

HEK-293T cells were transfected using the calcium phosphate method. ${ }^{28}$ Lentiviral particles were produced as previously described. ${ }^{29}$ MISSION pLKO.1-puro-based vectors encoded either a non-targeting shRNA (SHC002) or the following CDCA7 targeting shRNAs: sh-25 (TRCN0000140725), sh-40 (TRCN0000139240), sh-56 (TRCN0000139556) sh-83 (TRCN0000145183). DG-75, Ramos, BL2, Toledo, Molt-4 and S1F cell lines were transduced as described ${ }^{30}$ and selected in $1 \mu \mathrm{g} / \mathrm{ml}$ puromycin $>96 \mathrm{~h}$

\section{Antibodies}

The anti-CDCA7 S99 polyclonal rabbit serum was raised against the CRGRHPLPGSDSOSRRPR KLH-conjugated peptide as described..$^{31}$

\section{Cell proliferation and cell cycle analysis}

Cell proliferation was assessed by EdU incorporation as described. ${ }^{32}$ Cell cycle analysis was performed as previously described. ${ }^{30}$

\section{Transformation assays}

Transformation assays were performed as described. ${ }^{30}$ All mice were inoculated with control cells in one flank and CDCA7silenced cells in the opposite flank.

\section{Results}

\section{Transcriptomic analysis of immortalized and tumoral} cells

To identify genes participating in stages of malignant transformation beyond or independent of replicative immortalization, we compared the transcriptome of immortalized B cells (LCLs) and BL cell lines. Both cell types display similar growth, replication and viability rates when cultured in liquid media on plastic. ${ }^{30}$ LCLs cultured in liquid media grow mostly in clumps (Online Supplementary Figure S1). Single cells can also be found in these cultures, and most of them are anchored to the culture vessel surface, as indicated by their marked spreading 
(Online Supplementary Figure S1). As previously described, ${ }^{4}$ lymphoma cells form colonies in soft agar, whereas LCLs do not grow in this medium (Online Supplementary Figure S2). Together, these results suggest that anchorage to the vessel surface or to other cells might provide LCLs the signals required for cell proliferation and that soft agar might inhibit their growth by blocking anchorage to the surface and to neighboring cells.

A

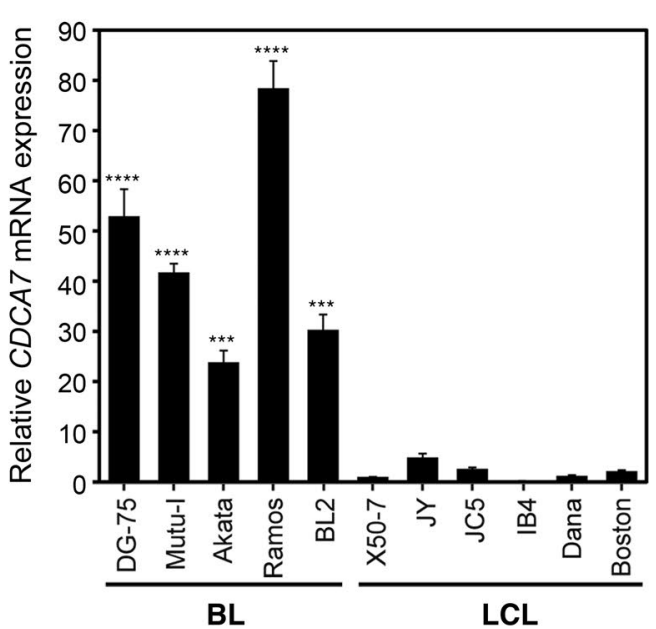

B
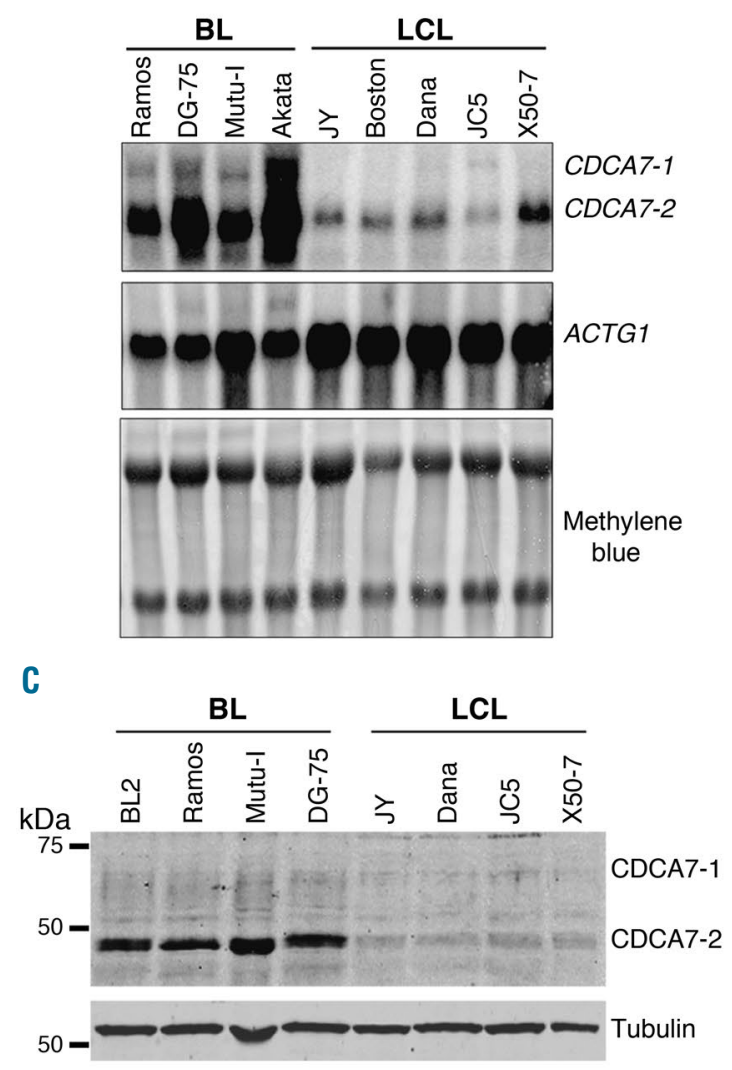

Figure 2. CDCA7 is overexpressed in BL cell lines. A. QPCR analysis of CDCA7 mRNA expression in the indicated $B L$ and $L C L$ cell lines. Expression is shown relative to that found in the $\mathrm{LCL}$ cell line IB4 and normalized by ACTB as mean+s.e.m. $(n=3)$. B. Northern blot analysis of CDCA7 isoforms and ACTG1 mRNA expression in the indicated BL and LCL cell lines. Staining of the membrane with methylene blue is also shown. C. Immunoblot analysis of CDCA7 expression in the indicated BL and LCL cell lines. Tubulin is shown as loading control.
A

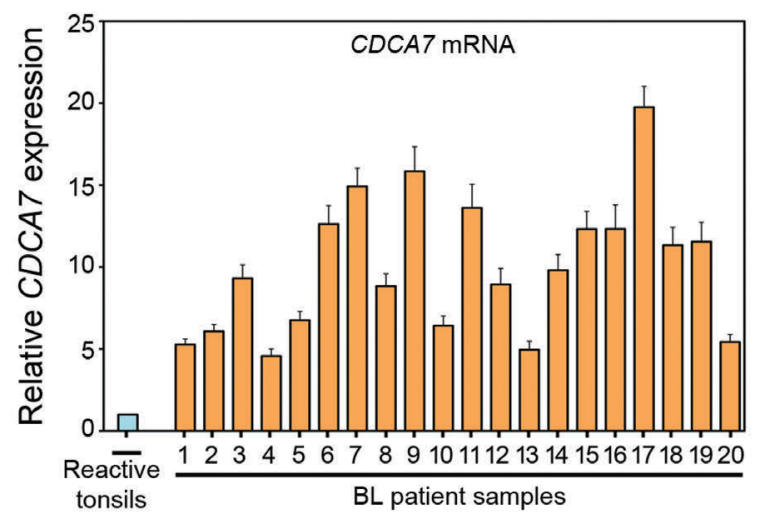

B

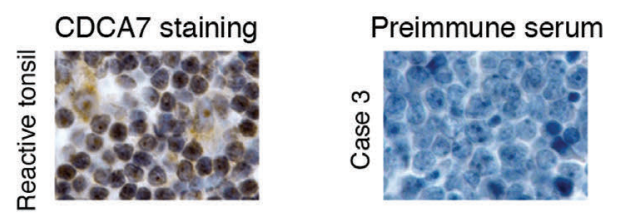

CDCA7 staining
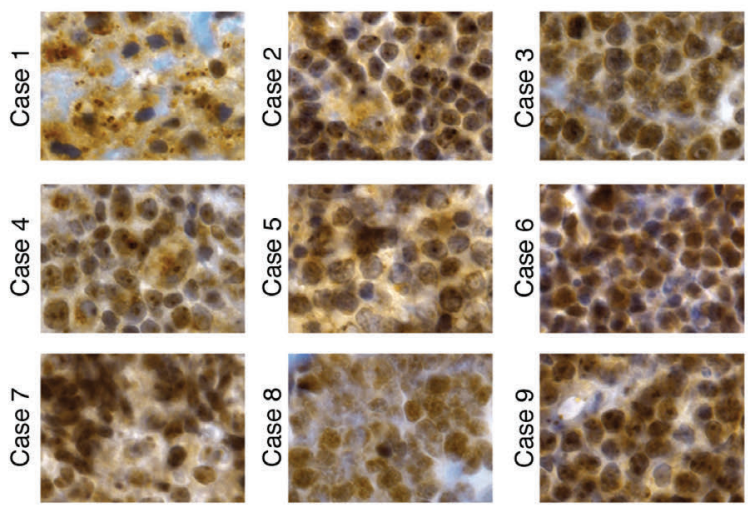

$\overline{10} \mu \mathrm{m}$

C

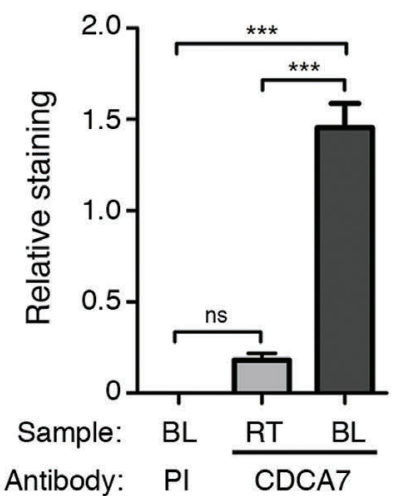

Figure 3. CDCA7 expression is deregulated in biopsies from BL patients. $A$ qPCR analysis of CDCA7 mRNA expression in a pool of 3 reactive tonsils and in $20 \mathrm{BL}$ patient samples. Data were normalized to the expression of ACTB and are shown relative to the pool of reactive tonsils as mean+s.e.m $(n=3)$. B. Sections from the germinal center of a reactive tonsil and from $9 \mathrm{BL}$ cases were analyzed by immunohistochemistry staining with anti-CDCA7 S99 antibody. A section of a $\mathrm{BL}$ case was also stained with the preimmune serum as negative control. Representative images are shown. C. Quantification of preimmune-positive area in immunohistochemistry-stained sections of $4 \mathrm{BL}$ cases (PI) and CDCA7-positive area in immunohistochemistry-stained sections of 4 reactive tonsils (RT) and $12 \mathrm{BL}$ cases. $* * * P<0.001$; one-way ANOVA with Bonferroni post-test. 
The comparison of the transcriptome of $5 \mathrm{BL}$ cell lines (DG-75, Ramos, BL2, Mutu-I and Akata) with a pool of 3 LCL cell lines (X50-7, IB-4 and Dana) using whole-genome microarrays showed $>1,600$ genes similarly expressed in $\mathrm{BL}$ cells that were up- (630 genes) or down-regulated $(1,033$ genes) significantly $(P<0.05)$ in $\mathrm{BL}$ cell lines (Figures $1 \mathrm{~A}-1 \mathrm{~B})$ (GEO accession number GSE41865). Among the genes that were regulated $>3$-fold (484 genes), we selected the 100 genes showing the highest statistical significance. Only 7 of these genes were up-regulated in BL cells (Figure 1C). The information known about the proteins encoded by these genes (http://www.uniprot.org) suggested that two of them (ID3 and CDCA7) might function as transcriptional regulators (Figure 1C) and, as such, they may have the potential to regulate gene expression programs related with malignant transformation. Indeed, ID3 is a critical mediator of BL formation. ${ }^{33,34}$ Since the role of CDCA7 in tumorigenesis was controversial, we investigated whether the elevated expression of CDCA7 might specifically play a critical role in the malignant transformation of lymphoid cells.

\section{CDCA7 expression is deregulated in Burkitt's Lymphoma}

There are two alternatively spliced CDCA7 isoforms (CDCA7-1 and CDCA7-2) that differ only in the presence of an additional internal exon in CDCA7-1 (Online Supplementary Figure S3A). CDCA7 mRNA levels, measured by GPCR using primers that amplify both isoforms, were markedly higher in BL than in LCL cells (Figure 2A). qPCR analysis with gene expression assays specific for each isoform and Northern blotting showed that CDCA72 was more abundant in both cell types and that levels of both isoforms were markedly higher in BL cells (Figure 2B and Online Supplementary Figure S3B).

CDCA7 protein levels were also compared between lymphoma and immortalized cells. Since we encountered reproducibility difficulties with commercially available antibodies (Abs), we generated a rabbit polyclonal $\mathrm{Ab}$ (S99) using a CDCA7 peptide present in both isoforms (Online Supplementary Figure S4A). To determine the specificity of this $\mathrm{Ab}$, we confirmed that it recognized CDCA71 and CDCA7-2 ectopically expressed in HEK-293T cells (Online Supplementary Figure S4B). Immunoblot analysis of BL and LCL cells using anti-CDCA7 S99 showed that CDCA7-2 levels were markedly higher in BL than in LCLs, whereas those of CDCA7-1 were barely detectable in both cell types (Figure 2C). CDCA7-2 was not detected in $\mathrm{BL}$ cell lysates if the $\mathrm{Ab}$ was previously neutralized with the peptide used during immunization (Online Supplementary Figure 3C), further demonstrating the specificity of the $\mathrm{S} 99$ anti-CDCA7 Ab.

To ascertain that the high expression of CDCA7 in BL cell lines was not just a consequence of their in vitro growth, we used qPCR to compare CDCA7 expression in biopsy specimens from $\mathrm{BL}$ patients with that in control tissues derived from reactive tonsils, which are enriched in germinal centre B cells. All BL samples expressed 5 to 20 times higher CDCA7 mRNA levels than a pool of 3 reactive tonsils (Figure $3 \mathrm{~A}$ ). Although reactive tonsils do not only contain B cells, the absence of additional cells is not expected to account for a $>5$-fold increase in CDCA7 mRNA expression in the tumor. In fact, CDCA7 protein immunostaining was also higher in BL samples than in the germinal centre of reactive tonsils (Figure $3 \mathrm{~B}$ ), a region enriched in B cells. Quantification of the CDCA7-positive area in immunohistochemistry-stained sections confirmed a marked expression increase in BL samples (Figure $3 \mathrm{C}$ ). Together, our results indicate that CDCA7 expression is

A

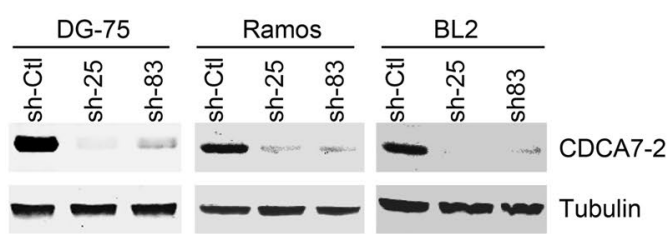

B

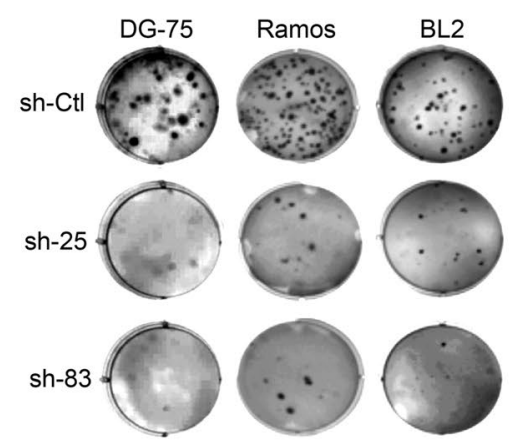

C
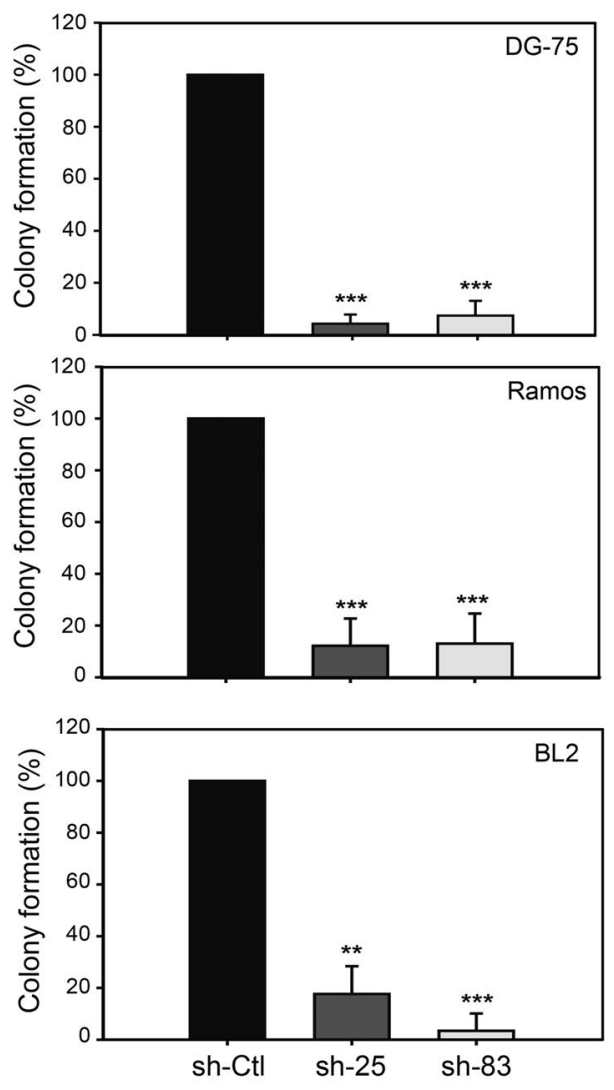

Figure 4. CDCA7 mediates anchorage-independent growth of BL cells. DG-75, $\mathrm{BL} 2$ and Ramos cells were transduced with lentivirus encoding sh-Ctl, sh-25 or sh- 83 and selected in the presence of puromycin $>5$ days. A. Representative CDCA7 immunoblot analysis of DG-75 $(n=4)$, Ramos $(n=5)$ and BL2 $(n=4)$ cells expressing the indicated shRNAs. Tubulin is shown as loading control. B. Representative images of wells containing these cells seeded in soft agar and C. colony formation quantification relative to cells expressing sh-Ctl shown as mean \pm s.e.m ( $n=4$ independent experiments, DG-75 and BL2; $n=5$ independent experiments, Ramos). ${ }^{*} P<0.01, * * * P<0.001$; one-way ANOVA with Bonferroni post-test. 
elevated in primary tumor samples derived from BL patients.

\section{CDCA7 mediates anchorage-independent growth of lymphoma cells}

The overexpression of CDCA7 in BL cells could be causally involved in oncogenesis or be only the result of a random event unrelated to tumor formation. To ascertain whether CDCA7 played a causative role in lymphomagenesis, we knocked-down its expression in BL tumor cells by using lentivirus encoding CDCA7-specific shRNA. By screening candidate shRNAs specific for CDCA7 in lymphoma cells, we identified sh-25 and sh-83 as having high knockdown capacity relative to a non-targeting shRNA (sh$\mathrm{Ctl}$ ) or to non-transduced cells (Online Supplementary Figure S5). Lentiviral transduction of DG-75 BL cells with sh-25 or sh-83 markedly inhibited CDCA7 expression relative to cells transduced with sh-Ctl (Figure 4A, left panel). CDCA7 silencing in these cells sharply decreased their colony formation capacity in soft agar (Figure 4B, left column; and Figure 4C, top panel). To determine whether CDCA7 plays a similar role in other $\mathrm{BL}$ cells, we transduced BL2 and Ramos BL cells with lentivirus encoding sh-25 or sh-83. These shRNAs efficiently silenced CDCA7 expression in these cells (Figure 4A, middle and right panels) and sharply decreased their growth in soft agar (Figure $4 \mathrm{~B}$, middle and right panels; and Figure $4 \mathrm{C}$, middle and bottom panels).
The inability of CDCA7-silenced cells to grow in soft agar may potentially be caused by inhibition of their capacity to grow under liquid culture conditions (anchorage-dependent growth). However, CDCA7 silencing in BL cells did not substantially affect their cell cycle distribution [Figure 5 (top panels) and Online Supplementary Figure S6] or inhibit their proliferation in liquid culture [Figure 5 (bottom panels) and Online Supplementary Figures $S 7 A-S 7 B]$. To investigate the potential role of CDCA7 in the regulation of cell proliferation by cell-cell contacts, we seeded single cells in 96-well plates and assessed their colony formation capacity. It should be noted that cells were able to attach to the surface of the wells under these conditions. As shown in Online Supplementary Figure S7C, CDCA7 knockdown did not decrease the number of colonies formed by DG-75 cells. Since BL is a very rapid growing tumor, it can be subjected to poor nutrient supply. To mimic nutrient shortage, we serum-starved DG75 cells and found that CDCA7 silencing did not affect the viability of cells cultured in the presence of low serum concentrations (Online Supplementary Figure S8A). CDCA7 knockdown also had no effect on the viability of cells treated with Cisplatin or Bleomycin (Online Supplementary Figure $S 8 B$ ).

Together, our data strongly support the notion that while CDCA7 is dispensable for anchorage-dependent growth, it is required for anchorage-independent growth
A
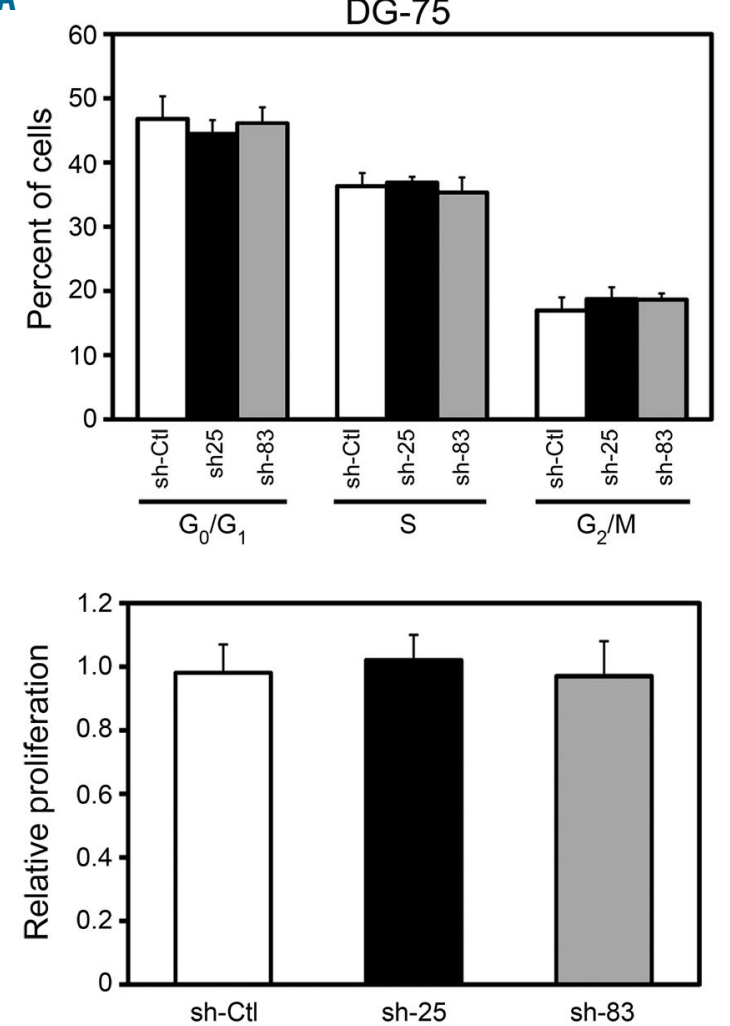

B
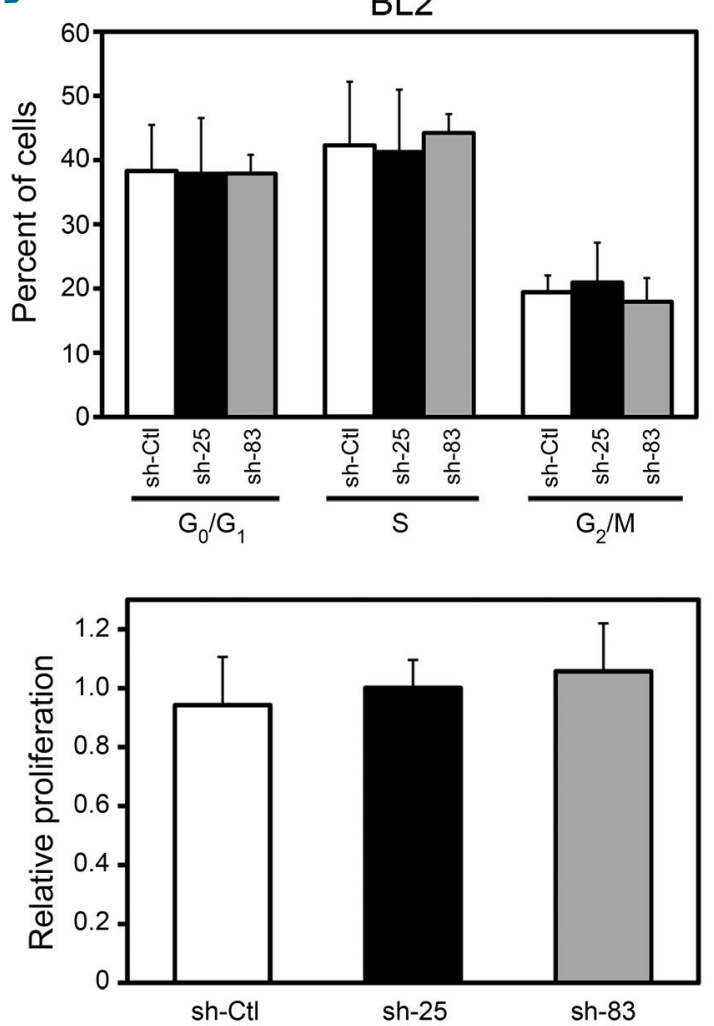

Figure 5. CDCA7 does not mediate anchorage-dependent growth. DG-75 and BL2 cells were transduced with lentivirus encoding sh-Ctl, sh-25 or sh-83 and selected in the presence of puromycin $>5$ days. Cell cycle and Edu incorporation analysis of (A) DG- 75 and (B) BL2 cells transduced with lentivirus encoding the indicated shRNAs. (Top panels) Columns show the percentage of each of these cells in the indicated cell cycle phases as mean \pm s.e.m ( $n=3$ ). (Bottom panels) Columns show normalized percentage of Edu incorporation in each of these cells as mean \pm s.e.m $(n=3)$. 
of lymphoma cells. However, high CDCA7 levels in immortal, but non-malignant $B$ cells, are not sufficient to promote anchorage-independent growth because forced expression of CDCA7-1 or CDCA7-2 in non-transformed JY cells or in JY cells transduced with lentivirus encoding constitutively active H-RAS (R12,T59) did not induce their growth in soft agar (Online Supplementary Figure S9). These results support the notion that malignant transformation requires the acquisition of multiple genetic lesions beyond those necessary to proliferate indefinitely.

A

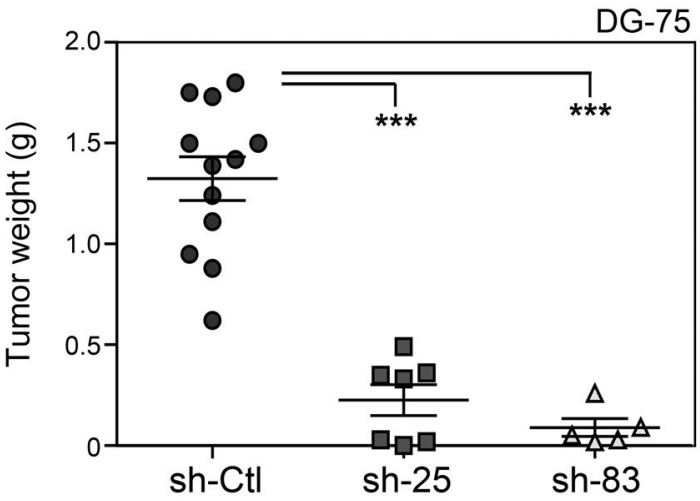

B

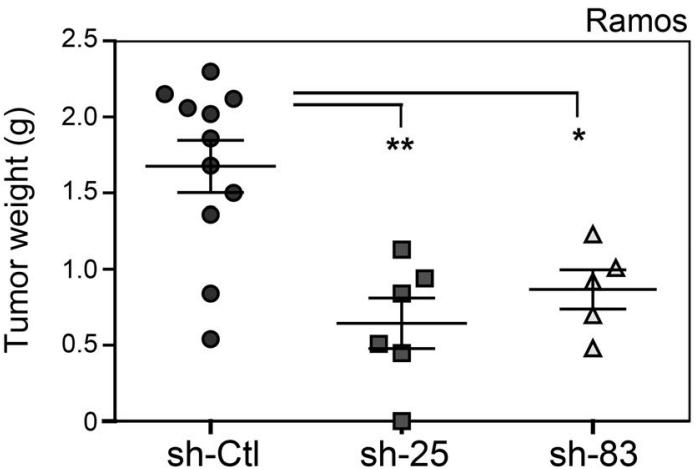

C

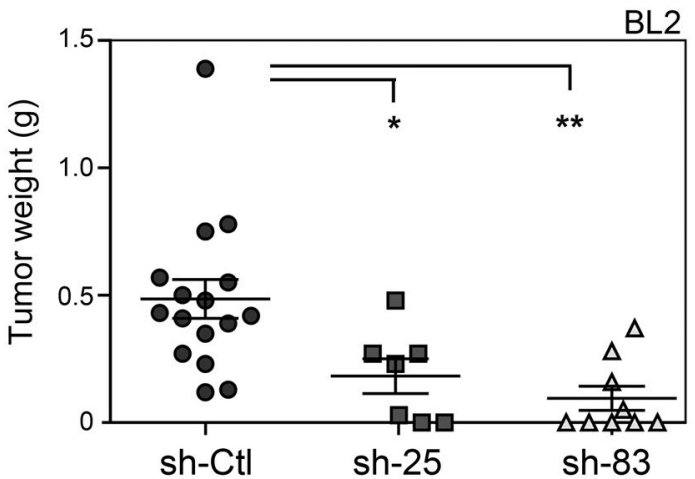

Figure 6. CDCA7 mediates BL tumor formation. DG-75, BL2 and Ramos cells were transduced with lentivirus encoding sh-Ctl, sh-25 or sh-83 and selected in the presence of puromycin $>5$ days. A. DG75, B. Ramos, and C. BL2 cells transduced with lentiviruses encoding the indicated shRNAs were inoculated subcutaneously in immunodeficient NOD-SCID mice. Tumors were extracted after 3 weeks. Circles, squares and triangles indicate the weight of individual tumors and horizontal bars indicate the mean (long bar) and s.e.m (short bar). $* P<0.05$, $* * P<0.01$ and $* * * P<0.001$ vs. sh-Ctl; one-way ANOVA with Bonferroni post-test.
CDCA7 mediates BL lymphoma growth in vivo

We have shown that DG-75, BL2, and Ramos BL cells are tumorigenic in immunodeficient mice, while EBV-immortalized B-cells are not. ${ }^{30,32}$ To investigate the contribution of CDCA7 to the in vivo tumorigenicity of BL cells, we transduced DG-75, BL2, and Ramos BL cells with sh-Ctl, sh-25 or sh- 83 and subcutaneously inoculated them in immunodeficient NOD-SCID mice. As expected, control-transduced DG-75, Ramos and BL cells readily elicited growth of large tumors in these mice (Figure 6). Importantly,
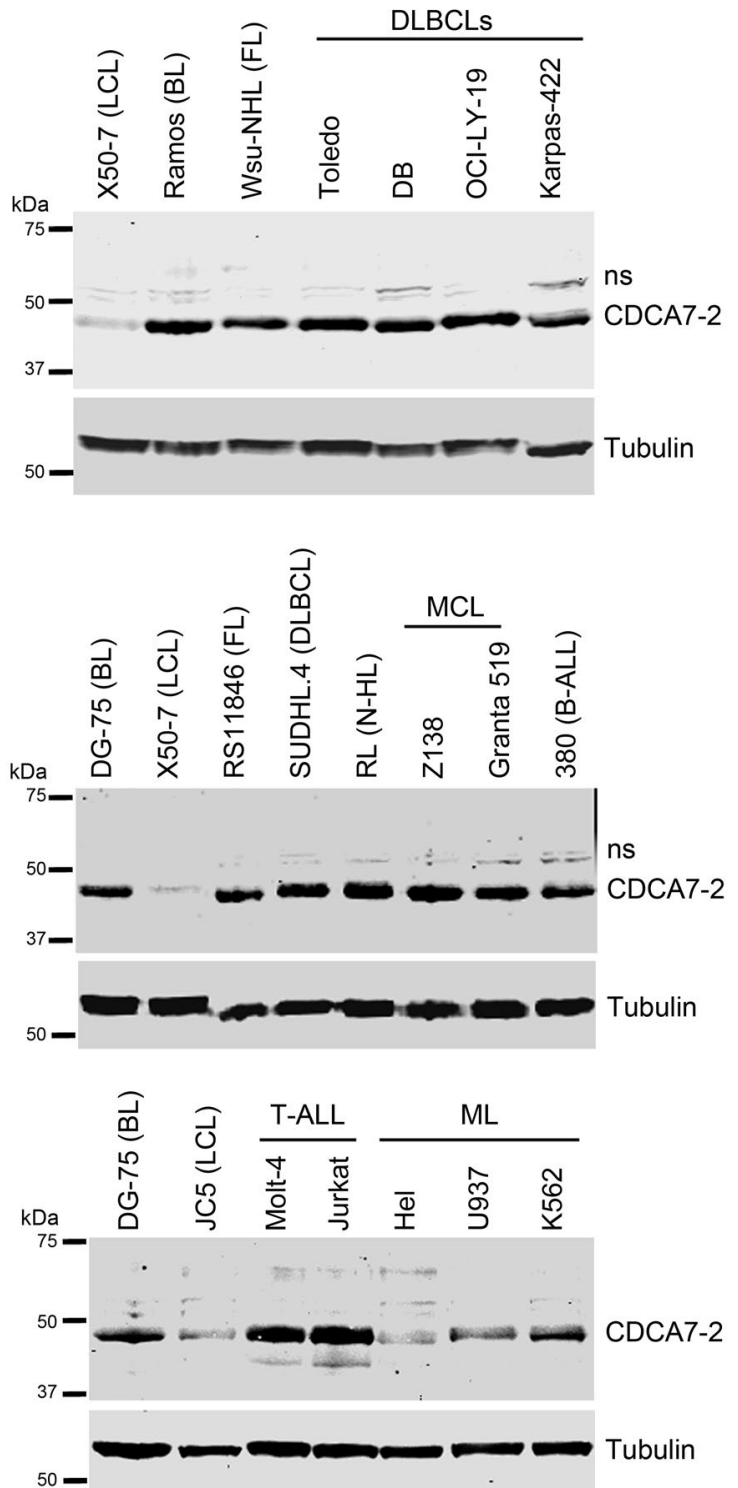

Figure 7. CDCA7 is overexpressed in various types of lymphoid malignancies Representative immunoblot analysis of CDCA7 expression in the indicated $\mathrm{BL}$, LCL, Diffuse Large B-Cell lymphoma (DLBCLs), Follicular Lymphoma (FL), Mantle Cell Lymphoma (MCL), uncharacterized non-Hodgkin Lymphoma (N-HL), Acute Lymphocytic B-cell leukemia (B-ALL) and T-cell leukemia (T-ALL), and Myeloid leukemia (ML) cell lines. Tubulin is shown as loading control. 
CDCA7 silencing in these cells blocked or greatly impaired their tumor formation capacity (Figure 6) without substantially affecting the expression of the Ki67 proliferation marker (Online Supplementary Figure S10). These results strongly suggest that CDCA7 is critical for BL formation and that therapies aimed at inhibiting its expression or its activity might be of interest for BL patients. Since current therapy for BL patients affects not only tumor cells but also actively proliferating normal cells from these patients, we investigated whether CDCA7 silencing affected the proliferation of primary human diploid fibroblasts IMR-90. Transduction of these cells with lentivirus encoding sh-25 or sh-83 decreased CDCA7 mRNA and protein levels relative to sh-Ctl-transduced cells (Online Supplementary Figures $S 11 A-S 11 B)$ without affecting their proliferation rate (Online Supplementary Figure S11C).

\section{CDCA7 expression is deregulated in lymphoid} neoplasias and mediates their growth in vivo

Next, we assessed whether CDCA7 is deregulated not only in $\mathrm{BL}$, but also in other types of cancer by comparing its expression in protein extracts of several lymphoid tumors. Cell lines of Diffuse Large B-cell Lymphoma (DLBCL), Follicular Lymphoma, Mantle Cell Lymphoma, uncharacterized non-Hodgkin Lymphoma, B-cell leukemia, and T-cell leukemia expressed markedly higher levels of CDCA7-2 than LCLs (Figure 7). In contrast, CDCA7-2 expression in myeloid leukemia cell lines was similar to that of LCLs (Figure 7, bottom panels).

To investigate whether CDCA7 is an essential mediator of lymphomagenesis not only in BL, but also in other lymphoid neoplasias, we silenced its expression in T-cell leukemia and DLBCL cells and analyzed their tumorigenicity in immunodeficient mice. Molt-4 T-cell leukemia and Toledo DLBCL cells expressed detectable levels of both CDCA7-1 and CDCA7-2 (Figure 8A) and their lentiviral transduction with sh-25 markedly inhibited the expression of both isoforms relative to cells transduced with sh-Ctl (Figure 8A). It should be noted that CDCA7-1 was not always detected in protein extracts from these cells. The capacity of these cells to form tumors was subsequently determined through their subcutaneous inoculation into immunodeficient mice. Molt-4 and Toledo cells transduced with sh-25 elicited formation of tumors significantly smaller than those produced by cells transduced with sh-Ctl (Figures 8B-8C). Similar to BL cells, CDCA7 silencing in Toledo cells did not substantially affect cell cycle distribution (Online Supplementary Figure S12). Together, our results strongly suggest that CDCA7 is a key mediator of lymphoid malignant transformation.

\section{Discussion}

In this study, through the identification of a gene involved in anchorage-independent growth, we found a new target for therapeutic intervention in lymphoid tumors less prone to cause side effects. By comparing gene expression profiles of immortal, but non-malignant cells, with those of tumor cells from the same lineage, we have uncovered a gene, CDCA7, whose elevated protein levels in lymphoid tumor cells mediate their anchorage-independent growth and their tumorigenesis without participating in their growth under normal tissue culture conditions (liquid culture on a rigid surface).
CDCA7 was initially identified as a MYC-responsive gene $^{19}$ whose expression can be transcriptionally induced also by E2F transcription factors ${ }^{22}$ and Notch. ${ }^{35}$ Although CDCA7 mRNA levels were shown to be upregulated in tumor samples relative to normal tissues, protein levels were not determined in these tumors. ${ }^{20}$ This is an important issue because the increase in mRNA expression does not necessarily imply an increase in protein content. Our results suggest that CDCA7 protein upregulation might be

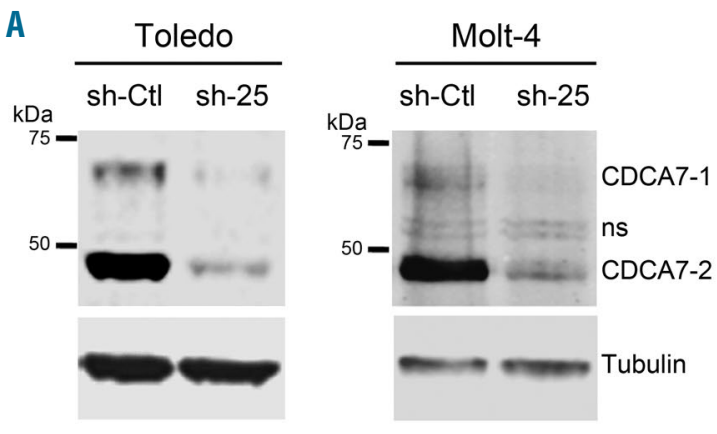

B
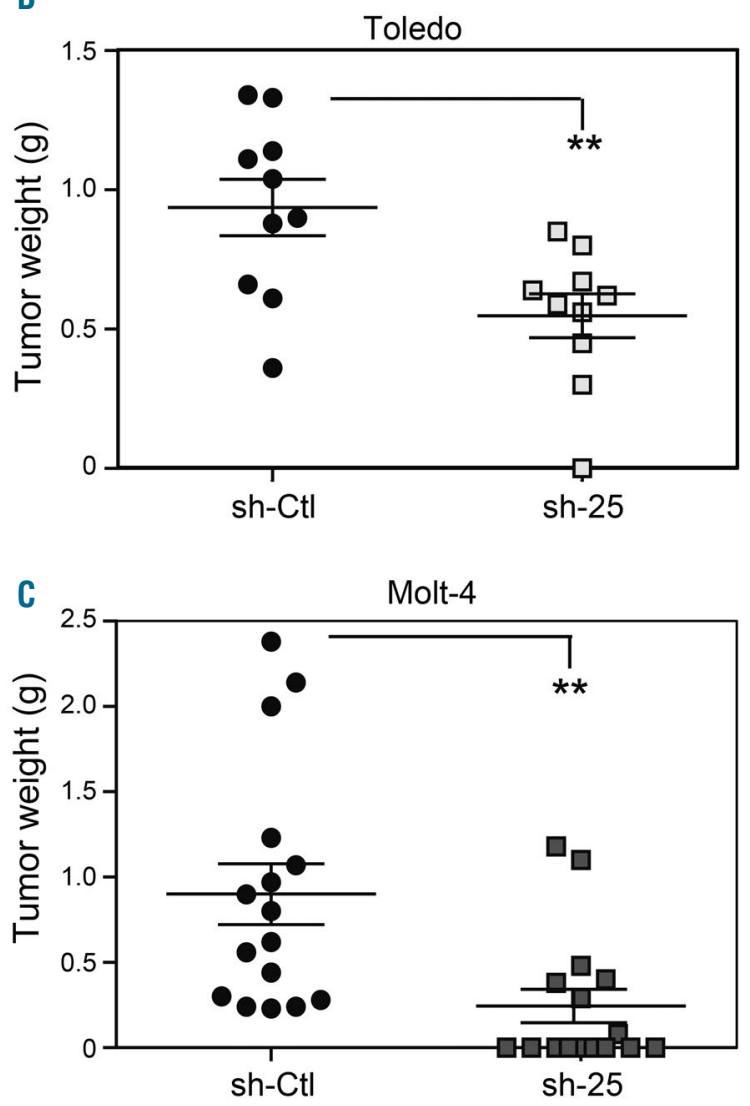

Figure 8. CDCA7 mediates tumorigenesis in T-cell leukemia and DLBCL cells. Toledo and Molt- 4 cells were transduced with lentivirus encoding sh-Ctl or sh- 25 and selected in the presence of puromycin $>5$ days. A. Representative CDCA7 immunoblot analysis of Toledo $(n=3)$, and Molt-4 $(n=3)$ cells expressing the indicated shRNAs. Tubulin is shown as loading control. B. Toledo and C. Molt-4 cells transduced with lentiviruses encoding the indicated shRNAs were inoculated subcutaneously in immunodeficient NOD-SCID mice. Tumors were extracted after 3 weeks. Circles and squares indicate the weight of individual tumors and horizontal bars indicate the mean (long bar) and s.e.m (short bar). ${ }^{*} P<0.01$ vs. sh-Ctl; paired samples t-test. 
a common event in lymphoid tumors. CDCA7 protein levels were not higher in myeloid tumors cell lines than in LCLs, but its expression in these tumors should be compared with that in control cells of the same lineage before drawing definitive conclusions. In addition, we cannot rule out that CDCA7 expression may be enhanced in other cell lines or in primary tumors.

MYC and activator E2F transcription factors are expressed only in proliferating cells, and there is a correlation between expression of their target genes and the growth rate of cells in culture. ${ }^{36}$ The increase of CDCA7 mRNA levels in tumor samples relative to control tissues could therefore have been merely the consequence of the presence of more proliferating cells in the tumor tissue. We have shown however that CDCA7 mRNA and protein levels are sharply elevated in lymphoid tumor cell lines relative to immortal cell lines (LCLs) and we had previously demonstrated that LCL and BL cell lines display indistinguishable proliferation rates and cell cycle profiles. ${ }^{30}$ These data suggest that the elevated expression of CDCA7 in lymphoid tumors is not simply the consequence of the presence of more proliferating cells relative to control tissues. Instead, our data support the notion that CDCA7 upregulation in these tumors is critical for anchorage-independent growth and tumorigenesis. Indeed, we show that its silencing in lymphoid tumor cells markedly inhibits their anchorage-independent growth and their tumor formation capacity in immunodeficient mice. These data therefore point to CDCA7 as a potential target for therapeutic intervention in lymphoid tumors.

Previous reports provided contradictive evidence for the role of CDCA7 in malignant transformation. While one report showed that CDCA7 overexpression in an immortal fibroblast cell line impaired MYC-induced colony formation, ${ }^{21}$ other reports proposed that forced CDCA7 expression promoted transformation in vitro and in vivo., ${ }^{190}$ However, the experimental evidence supporting a transforming activity for CDCA7 was very weak. On the one hand, CDCA7 overexpression in a B-cell line or in a fibroblast cell line increased only modestly their already existing capacity to form colonies in soft agar. ${ }^{19}$ Since both cell lines had a basal capacity to grow in this semisolid medium, it seems that these cells, instead of being merely immortal, were already transformed or at least partially transformed. On the other hand, only 4 out of $45(8.88 \%)$ transgenic mice overexpressing CDCA7 in the B-cell compartment presented lymphoid malignancies at 1 year, whereas 3 out of $28(10.71 \%)$ control littermates presented lymphomas. ${ }^{20}$ Together, these data did not support the notion that CDCA7 overexpression induces malignant transformation. In fact, we have shown herein that CDCA7 overexpression in non-transformed $\mathrm{B}$ cells fails to induce their growth in soft agar even in combination with constitutively active $\mathrm{H}$ RAS. Our data therefore suggest that CDCA7 is necessary but not sufficient for lymphoid malignant transformation.
Numerous genes that mediate tumor transformation and anchorage-independent growth are also essential for cell proliferation over rigid surfaces. This is the case, for instance, of E2F1, whose knock-down in BL cells inhibits tumor growth in vivo, colony formation in soft agar, and cell proliferation in liquid culture. ${ }^{30}$ The absence of anchorage of non-tumor cells to a rigid surface does not permit their proliferation likely because they miss either the growth- or the survival-promoting signals provided by anchorage, ${ }^{37}$ whereas tumor cells might autonomously generate these signals. ${ }^{37}$ While normal epithelial cells and fibroblasts require attachment to a rigid surface for survival, lymphocytes are viable in the blood and lymph. However, normal lymphocytes do not proliferate in the blood, but only in lymphoid organs where they might potentially interact with the extracellular matrix and with other cells within these organs. Thus, it seems likely that deprivation of growth-promoting signals provided by anchorage accounts for the inability of non-tumor lymphoid cells to proliferate in soft agar. Aberrantly elevated CDCA7 levels in lymphoid tumor cells might correspondingly contribute to generate the growth-promoting signals provided by anchorage. It will be therefore of great interest to investigate the molecular mechanisms involved in CDCA7-promoted anchorage-independent growth.

Together, our findings identify CDCA7 as an important regulator of lymphoid tumor transformation. The inhibition of tumor formation capacities of T-cell leukemia, DLBCL, and BL cells upon CDCA7 silencing point to CDCA7 as a candidate for therapeutic intervention in lymphoid tumors. Since CDCA7 knock-down in primary diploid fibroblasts does not inhibit their proliferation, it seems plausible that therapies aimed at inhibiting CDCA7 expression or function might significantly inhibit the growth of lymphoid tumors while likely not affecting the proliferation of normal cells.

\section{Acknowledgments}

The authors would like to thank D. Trono for plasmids; the CNIO Tumor Bank for providing some of the cases included in this study; and the Bioinformatics facility of Centro Nacional de Investigaciones Cardiovasculares (Madrid, Spain) for analysis of microarray data.

\section{Funding}

This work was supported by the Spanish Ministerio de Economia, Industria y Competitividad (MEIC) grants to MRC. (SAF2013-45258P and SAF2017-88881-R) and TIV. (SAF2014-52737P); and by Instituto de Salud Carlos III (CIBERNED) to TIV. The cost of this publication has been paid in part with FEDER funds. OK holds an FPI fellowship from MEIC (BES-2014-069236). The CNIC is supported by the Ministerio de Ciencia, Investigacion y Universidades and the Pro CNIC Foundation, and is a Severo Ochoa Center of Excellence (SEV-2015-0505).

\section{References}

1. Hanahan D, Weinberg RA. The hallmarks of cancer. Cell. 2000;100(1):57-70.

2. Hanahan D, Weinberg RA. Hallmarks of cancer: the next generation. Cell. 2011; 144(5):646-674.

3. Jainchill JL, Aaronson SA, Todaro GJ.
Murine sarcoma and leukemia viruses: assay using clonal lines of contact-inhibited mouse cells. J Virol. 1969;4(5):549-553.

4. Nilsson K, Giovanella BC, Stehlin JS, Klein G. Tumorigenicity of human hematopoietic cell lines in athymic nude mice. Int J Cancer. 1977;19(3):337-344.

5. Epstein MA, Achong, B. G. The relationship of the virus to Burkitt's lymphoma. In:
Epstein M.A. ABGe, ed. The Epstein-Barr Virus. Berlin, Heidelberg: Springer, 1979.

6. Discher DE, Janmey P, Wang YL. Tissue cells feel and respond to the stiffness of their substrate. Science. 2005; 310(5751): 1139-1143.

7. Otsuka H, Moskowitz M. Arrest of 3T3 cells in G1 phase in suspension culture. J Cell Physiol. 1975;87(2):213-219. 
8. Hecht JL, Aster JC. Molecular biology of Burkitt's lymphoma. J Clin Oncol. 2000; 18(21):3707-3721.

9. Boxer LM, Dang CV. Translocations involving c-myc and c-myc function. Oncogene. 2001;20(40):5595-5610.

10. Cole MD, McMahon SB. The Myc oncoprotein: a critical evaluation of transactivation and target gene regulation. Oncogene. 1999;18(19):2916-2924.

11. Dang CV. MYC on the path to cancer. Cell. 2012;149(1):22-35

12. Grandori C, Cowley SM, James LP, Eisenman RN. The Myc/Max/Mad network and the transcriptional control of cell behavior. Annu Rev Cell Dev Biol. 2000; 16:653-699.

13. Nesbit CE, Tersak JM, Prochownik EV. MYC oncogenes and human neoplastic disease. Oncogene. 1999;18(19):3004-3016.

14. Pelengaris S, Khan M, Evan G. c-MYC: more than just a matter of life and death. Nat Rev Cancer. 2002;2(10):764-776.

15. Chung HJ, Levens D. c-myc expression: keep the noise down! Mol Cells. 2005;20(2):157-166.

16. Adams JM, Harris AW, Pinkert CA, et al. The c-myc oncogene driven by immunoglobulin enhancers induces lymphoid malignancy in transgenic mice. Nature. 1985;318(6046):533-538.

17. Felsher DW, Zetterberg A, Zhu J, Tlsty T, Bishop JM. Overexpression of MYC causes p53-dependent G2 arrest of normal fibroblasts. Proc Natl Acad Sci USA. 2000; 97(19):10544-10548.

18. Packham G, Cleveland JL. c-Myc and apoptosis. Biochim Biophys Acta. 1995; 1242(1):11-28

19. Prescott JE, Osthus RC, Lee LA, et al. A novel c-Myc-responsive gene, JPO1, participates in neoplastic transformation. J Biol Chem. 2001;276(51):48276-48284
20. Osthus RC, Karim B, Prescott JE, et al. The Myc target gene JPO1/CDCA7 is frequently overexpressed in human tumors and has limited transforming activity in vivo. Cancer Res. 2005;65(13):5620-5627.

21. Gill RM, Gabor TV, Couzens AL, Scheid MP. The MYC-associated protein CDCA7 is phosphorylated by AKT to regulate MYC-dependent apoptosis and transformation. Mol Cell Biol. 2013;33(3):498-513.

22. Goto $Y$, Hayashi R, Muramatsu T, et al JPO1/CDCA7, a novel transcription factor E2F1-induced protein, possesses intrinsic transcriptional regulator activity. Biochim Biophys Acta. 2006;1759(1-2):60-68

23. Thijssen PE, Ito Y, Grillo G, et al. Mutations in CDCA7 and HELLS cause immunodeficiency-centromeric instability-facial anomalies syndrome. Nat Commun. 2015 6:7870.

24. Jenness C, Giunta S, Muller MM, Kimura H, Muir TW, Funabiki H. HELLS and CDCA7 comprise a bipartite nucleosome remodeling complex defective in ICF syndrome. Proc Natl Acad Sci USA. 2018; 115(5):E876-E885.

25. Whitfield ML, Sherlock G, Saldanha AJ, et al. Identification of genes periodically expressed in the human cell cycle and their expression in tumors. Mol Biol Cell. 2002;13(6):1977-2000.

26. Esteban V, Mendez-Barbero N, JimenezBorreguero LJ, et al. Regulator of calcineurin 1 mediates pathological vascular wall remodeling. I Exp Med. 2011; 208(10):2125-2139

27. Campanero MR, Herrero A, Calvo V. The histone deacetylase inhibitor trichostatin $\mathrm{A}$ induces GADD45 gamma expression via Oct and NF-Y binding sites. Oncogene. 2008;27(9):1263-1272.

28. Campanero MR, Armstrong M, Flemington E. Distinct cellular factors regulate the c- myb promoter through its E2F element Mol Cell Biol. 1999;19(12):8442-8450.

29. Alvaro-Blanco J, Urso K, Chiodo Y, et al MAZ induces MYB expression during the exit from quiescence via the E2F site in the MYB promoter. Nucleic Acids Res. 2017;45(17):9960-9975

30. Molina-Privado I, Rodriguez-Martinez M Rebollo P, et al. E2F1 expression is deregulated and plays an oncogenic role in sporadic Burkitt's lymphoma. Cancer Res. 2009;69(9):4052-4058.

31. Alvaro-Blanco J, Martinez-Gac L, Calonge E, et al. A novel factor distinct from E2F mediates C-MYC promoter activation through its E2F element during exit from quiescence. Carcinogenesis. 2009; 30(3): 440-448.

32. Molina-Privado I, Jimenez PR, MontesMoreno S, et al. E2F4 plays a key role in Burkitt lymphoma tumorigenesis. Leukemia. 2012;28(10):2277-2285.

33. Richter J, Schlesner M, Hoffmann S, et al. Recurrent mutation of the ID3 gene in Burkitt lymphoma identified by integrated genome, exome and transcriptome sequencing. Nat Genet. 2012;44(12):13161320

34. Love C, Sun Z, Jima D, et al. The genetic landscape of mutations in Burkitt lymphoma. Nat Genet. 2012:44(12):1321-1325.

35. Guiu J, Bergen DJ, De Pater E, et al Identification of Cdca7 as a novel Notch transcriptional target involved in hematopoietic stem cell emergence. J Exp Med. 2014;211(12):2411-2423.

36. Ross DT, Scherf U, Eisen MB, et al Systematic variation in gene expression patterns in human cancer cell lines. Nat Genet. 2000;24(3):227-235.

37. Wolfenson H, Bershadsky A, Henis YI Geiger B. Actomyosin-generated tension controls the molecular kinetics of focal adhesions. J Cell Sci. 2011;124(Pt 9):1425-1432. 